\title{
Multilevel Analysis of Factors Associated with Stunting Among Under Five Children in Ethiopia
}

\author{
Hulle Hassen* Kemal Nure \\ College of Natural and Computational Science, Madda Walabu University, Bale Robe, Ethiopia
}

Introduction: Stunting is a major health problem in children under-five years in many low and middle income countries around the world. This study was aimed to identify factors associated with stunting among under five age children in Ethiopia.

Methods: Cross-sectional data from Ethiopian Demographic and Health Survey was used for the analysis. The statistical models that suit the hierarchical data such as variance components model, random intercept model, and random coefficients model were used to analyze the data.

Results: This study revealed that among the under-five children considered in the study around $37.1 \%$ children was stunted. Age of children, region, place of residence, wealth index, mothers BMI, incidence of diarrhea in the last two weeks before survey and mother and husband/partner educational level were found to be significant predictors for stunting.

Conclusions: Multilevel logistic regression shows that there is heterogeneity or cross-regional variation in stunting. Further this model implies that there exist considerable differences in stunting among regions and a model with a random coefficient is more appropriate to explain the regional variation than a model with fixed coefficients or empty model with random effects.

Keywords: Multilevel model, under five children; Stunting

DOI: $10.7176 / \mathrm{FSQM} / 89-01$

Publication date: August 31st 2019

\section{Introduction}

Malnutrition indicators are caused by an extremely low energy and protein intake, nutrients losses due to infection or combination of both low energy/ protein intake and high nutrient loss by the mother during pregnancy or by the child after birth (WHO, 2000).

Worldwide, over 10 million children under the age of 5 years die every year from preventable and treatable illnesses despite effective health interventions (Mussie A. et al., 2014). In developing countries, malnutrition is a major health problem (Caulfield et al., 2004). Childhood stunting is one of the most significant impediments to human development, globally affecting approximately 162 million children under the age of five years. Stunting, or being too short for one's age, is defined as a height that is more than two standard deviations below the WHO child growth standards median (WHO, 2006). Stunting is a major health problem in children under five years in many low and middle income countries around the world (UNICEF, 2015). It is defined as a deficit in height relative to a child's age (De Onis $\mathrm{M}$. WHO, 2006).

Stunting has long term effects on individuals and societies including diminished cognitive and physical development reduced productive capacity, poor health and an increased risk of degenerative diseases such as diabetes (The state of the world's children, 2013). If current trends continue, projections indicate that 127 million children under five years will be stunted in 2025 (Walker et al., 2007). An estimated 80\% of world's stunted children lived in just fourteen countries (India, Nigeria, China, Pakistan, Indonesia, Bangladesh, Ethiopia, Democratic Republic of Congo, Philippines, United Republic of Tanzania, Egypt, Kenya, Uganda and Sudan). Sub-Saharan Africa and South Asia were the home to three fourths of the world's stunted children $40 \%$ and $39 \%$, respectively (Desalegne et al., 2016).

In Africa, high prevalence levels of stunting among children under-five years of age (36\% or 56 million in 2011). Africa shows rising numbers of stunted children due to population increase and an almost stagnant prevalence of stunting over the past two decades of the 34 countries that account for $90 \%$ of the global burden of malnutrition, 22 are in Africa. Some African countries (e.g. Ethiopia, Ghana and Mauritania) have had substantial reductions in stunting but overall in this region little improvement is anticipated in the coming years if recent trends continue (De Onis M., 2006). In Africa, an estimated 13.4 million children under-five years of age or $8.5 \%$ were wasted $(\mathrm{W} / \mathrm{H}<-2 \mathrm{SD})$ in 2011 . These children are at substantial increased risk of death. Increasing trends in child overweight in most world regions not just the developed world. In Africa, the estimated prevalence underfive overweight increased from $4 \%$ in 1990 to $7 \%$ in 2011.

According to CSA report in 2011 EDHS nationally 44 percent of children under age five are stunted and 21 percent of children are severely stunted. Male children are slightly more likely to be stunted than female children (46 percent and 43 percent respectively). 
Objectives of the study

The main concerns of authors were to identify the major factors associated with stunting among under five age children in Ethiopia., that is,

(i) to analyze the within- and between-regions variation of stunting among under five age children in Ethiopia.

(ii) to make model comparison and suggest an appropriate model for analyzing stunting among under five age children in Ethiopia.

(iii) to identify the most important socio-economic, demographic and environmental factors associated with stunting level among under-five children in Ethiopia.

\section{Data and Methodology}

The source of data for this study was the 2011 Ethiopia Demographic and Health Survey (EDHS) which is obtained from Central Statistical Agency (CSA). The study populations are all the under five children residents of Ethiopia using the 2011 EDHS data set. In the 2011 EDHS from 11,654 under-five children, total number of children covered in the current study on the stunting status of children is based on 9370 under-five children with complete anthropometric measurements and the study considered height-for-age anthropometric index as indicator of a children's stunting status respectively.

\section{Variables in the Study Dependent variable}

Binary and multi category outcomes are very common in biomedical studies, for instance in the evaluation of nutritional status among children of under-five. Based on these classifications, it is possible to employ plausible statistical tools for estimating the magnitude of the association between the response variable of interest as a function of predictor variables.

$$
\text { stunted }=\left\{\begin{array}{l}
1 \quad \text { if stunted }(z-\text { score }<-2) \\
0 \text { if not stunted }(z-\text { score } \geq-2)
\end{array}\right\}
$$

\section{Independent variables}

The explanatory variables included in this study are mother's education, employment status of the mother, education of husband/partner, household income, household size, place of residence and geographical region, age of the child, sex of the child, birth interval, birth order of the child's, diarrhea and fever in the last two weeks before survey, water supplies and toilet facilities, Incidence of acute respiratory infection (such as cough) in the last two weeks, Mother's nutritional status or Mother's BMI are important factors are included in this study.

\section{Multilevel Logistic Regression Model}

Multilevel logistic regression model as a hierarchical model, can account for lack of independence across levels of nested data (i.e., individuals nested within groups). Let $y_{i j}$ be the binary outcome variable, coded ' 0 ' or ' 1 ', associated with level-one unit $i$ nested within level two unit $j$. Also let $\mathrm{p}_{i j}$ be the probability that the response variable equals 1 , and $\mathrm{p}_{i j}=\operatorname{Pr}(\mathrm{y} i j=1)$. Here, $\mathrm{y}_{i j}$ follows a Bernoulli distribution. Like the logistic regression the $\mathrm{p}_{i j}$ is modeled using the link function, logit. The two-level logistic regression model can be written as,

$$
\log \left(\frac{p_{i j}}{1-p_{i j}}\right)=\beta_{0}+\beta_{1} x_{i j}+u_{o j}
$$

Where $u_{j}$ is the random effect at level two. Without $u_{j}$, this equation (3.11) can be considered as a standard logistic regression model. Therefore, conditional on $u_{j}$, the $y_{i j}$ 's can be assumed to be independently distributed. Here, $u_{j}$ is a random quantity and follows $\mathrm{N}\left(0, \delta_{u}^{2}\right)$.

Equivalently, we can split model (3.11) into two models: one for level 1 and the other for level 2.

$$
\begin{array}{ll}
\log \left[\frac{p_{i j}}{1-p_{i j}}\right]=\beta_{0 j}+\beta_{1} x_{i j} & \text { model: level } 1 \\
\text { And } \beta_{0 j}=\beta_{0}+u_{o j} & \text { model: level } 2
\end{array}
$$

\section{Testing Heterogeneity of Proportions}

For the proper application of multilevel analysis in general and multilevel logistic regression analysis in particular, the first logical step is to test for heterogeneity of proportions between groups (in our case between Regions). To test whether there are indeed systematic differences between groups, the well-known chi-square test for contingency table can be used. In this case the chi-square test statistic is: 


$$
x^{2}=\sum_{j=1}^{N} n_{j} \frac{\left(p_{j}-\hat{p} .\right)}{\hat{p}(1-\hat{p} .)}
$$

\section{Variance Components Model}

The empty two-level model for a dichotomous outcome variable refers to a population of groups (level-two units (regions)) and specifies the probability distribution for group-dependent probabilities $\pi_{i j}$ in $y_{i j}=p_{i j}+\varepsilon_{i j}$ in without taking further explanatory variables into account. For the logit link function, the log-odds have a normal distribution in the population of groups, which is expressed by

$\log i t\left(p_{j}\right)=\beta_{0}+U_{0 j}$

And the probability corresponding to the average value $\beta_{0}$, denoted by $\pi_{0}$

$\pi_{0}=\frac{\exp \left(\beta_{0}\right)}{1+\exp \left(\beta_{0}\right)}$

\section{The Random Intercept Model}

In the random intercept model the intercept is the only random effect meaning that the groups differ with respect to the average value of the response variable. But the relation between explanatory variables and the response can differ between groups in more ways.

The random intercept model expresses the logit of $p_{i j}$ as a sum of a linear function of the explanatory variables.

That is,

$$
\begin{aligned}
& \log i t\left(p_{i j}\right)=\log \left(\frac{p_{i j}}{1-p_{i j}}\right)=\beta_{0 j}+\beta_{1} x_{1 i j}+\beta_{2} x_{2 i j}+\ldots+\beta_{k} x_{k i j} \\
& \beta_{0 j}=\beta_{0}+u_{o j} \text { As a result } \\
& \log i t\left(p_{i j}\right)=\log \left(\frac{p_{i j}}{1-p_{i j}}\right)=\beta_{0}+\beta_{1} x_{1 i j}+\beta_{2} x_{2 i j}+\ldots+\beta_{k} x_{k i j}+U_{0 j}
\end{aligned}
$$

Where the intercept term $\beta_{0 j}$ is assumed to vary randomly and is given by the sum of an average intercept $\beta_{0}$ and group-dependent deviations, $U_{0 j}$. The first part incorporating the regression coefficients is the fixed part of the model, because the coefficients are fixed. The remaining part $U_{0 j}$ is called the random part of the model. It is assumed that the residual, $U_{0 j}$ are mutually independent and normally distributed with mean zero and variance $\delta_{u}^{2}$. Thus, a unit difference between the $x_{h}$ values of two individuals in the same group is associated with a difference of $\beta_{h}$ in their log-odds, or equivalently, a ratio of $\exp \left(\beta_{h}\right)$ in their odds.

\section{The Random Coefficient Model}

In above we have allowed the probability of stunting and wasting to vary across regions, but we have assumed that the effects of the explanatory variables are the same for each region. Now modify this assumption by allowing the difference between explanatory variables within a region to vary across regions. To allow for this effect, we will need to introduce a random coefficient for those explanatory variables. So, a random coefficient model represents heterogeneity in relationship between the response and explanatory variables. Suppose that there is k level-one explanatory variables $\mathrm{X}_{1}, \mathrm{X}_{2} \ldots \mathrm{X}_{\mathrm{k}}$ and consider the model where all predictor variables have varying slopes and random intercept. That is

$\log i t\left(p_{i j}\right)=\log \left(\frac{p_{i j}}{1-p_{i j}}\right)=\beta_{0 j}+\beta_{1 j} x_{1 i j}+\beta_{2 j} x_{2 i j}+\ldots+\beta_{k j} x_{k i j}$

Letting $\beta_{0 j}=\beta_{0}+U_{o j}$ and $\beta_{h j}=\beta_{h}+U_{h j}$ where $\mathrm{h}=1,2, \ldots \mathrm{k}$, we have: 
$\log \left(p_{i j}\right)=\log \left(\frac{p_{i j}}{1-p_{i j}}\right)=\beta_{o}+\sum_{h=1}^{k} \beta_{h} X_{i j}+U_{o j}+\sum_{h=1}^{k} U_{h j} X_{i j}$

The first part $\beta_{o}+\sum_{h=1}^{k} \beta_{h} X_{i j}$ is called the fixed part of the model and the second part $U_{o j}+\sum_{h=1}^{k} U_{h j} X_{i j}$ is called the random part of the model. The random variables or effects $U_{o j}, U_{1 j}, \ldots, U_{k j}$ are assumed to be independent between groups but may be correlated within groups.

\section{Model Selection Criteria}

To achieve this task selection criterion's such as R-square, adjusted R-square, Pseudo $\mathrm{R}^{2}$, BIC, AIC, etc should be considered. In the case of logistic regression, the model selection criteria will be taken as AIC. The AIC computation is based on the likelihood of the fit and the number of parameters in the model is considered.

\section{Test of overall model fit}

For the selected model before proceeding to examine the individual coefficients, we should look at an overall test of the null hypothesis that the location coefficients for all of the variables in the model are 0 . It can base this on the change in -2 log-likelihood when the variables are added to a model that contains only the intercept. The change in likelihood function has a chi-square distribution even when there are cells with small observed and predicted counts. This value provides a measure of how well the model fits the data. The log likelihood statistic is analogous to the error sum of squares in multiple linear regressions. As such it is an indicator of how much unexplained information remains after fitting the model. The larger the value of the log likelihood the more unexplained observations there are and a poorly fitting model. Therefore, a good model means a small value for $-2 \mathrm{LL}$. If a model fits perfectly, the likelihood is 1 and $-2 \times \log 1=0$ (Agresti, 2002).

The likelihood-ratio test statistic is given by (Hosmer and Lemeshow, 2000)

$G^{2}=-2 \ln \left(\frac{\text { likelihood without the variable }}{\text { likelihood with the variable }}\right)$

\section{Results}

The analysis was done using SPSS version 20 and STATA version 12.

\section{Descriptive statistics}

Table 1 shows that the relative frequency distributions of the stunting status of child. $37.1 \%$ are stunted and $62.9 \%$ are not stunted.

\section{Result of Multilevel Logistic Regression Analysis}

The first step in performing a multilevel analysis is testing the heterogeneity of proportions between groups (regions). For stunting chi-square test statistic was applied to assess heterogeneity in the proportion of individuals among regions. The test yield $\chi^{2}(10)=280.4339$ with $\mathrm{P}=0.000<0.05$, where 10 is the degrees of freedom. Thus, there is an evidence of heterogeneity of individuals among regions. For wasting $\chi^{2}(10)=141.8441 \mathrm{P}=0.000$ which is less than 0.05 indicating that there is heterogeneity among region.

\section{Variance components model}

This is the type of model that incorporates only the grand mean and random intercept (regional effect) without covariate.

Table 2 shows the output of the estimates of fixed effects and random effects. From the table we can see that the estimate of the fixed part of the model is -0.669 with $z$-value of -4.71 and p-value of 0.000 which implies that the average $\log$ odd of stunting is significantly different from zero. The intercept informs us $\hat{\beta}_{o}=-0.669$ that the average probability of stunting is $\frac{\exp (-0.669)}{1+\exp (-0.669)}=0.339$ which means the chance of stunting is $33.9 \%$ on average.

The table also contains the variance estimate of random effects at regional level, with confidence interval of $(0.0887,0.5215)$ which implies that the between region variance of stunting is 0.2150 and reveals that there is a significant difference in stunting among children across regions. This implies that multilevel model is more appropriate relative to single level. At the bottom of the table there is the result of the hypothesis $\mathrm{H}_{0}: \sigma^{2} u=0$ is provided showing that there is no cross-regional variation in stunting. For this hypothesis, we see that the value of the test statistic is 240.42 with $p=0.0000$ Therefore, the null hypothesis is rejected and there is evidence of heterogeneity or cross-regional variation in stunting.

\section{Random Intercept and fixed coefficient logistic regression analysis}


In a random intercept and fixed coefficient multilevel logistic regression model, we allowed the probability of stunting to vary across regions, but we assumed that the effects of the explanatory variables are the same for each region. That is, the random intercept varies across regions, but children level explanatory variables are fixed across regions.

The Wald test of overall goodness of fit gives Wald chi2 $(12)=609.44$ with $p=0.0000$ where 12 is the degrees of freedom. This indicates that all explanatory variables jointly are significant. From the table we see that the inclusion of level one covariates decreased regional variations from 0.2150 (level-two variance without covariates) to .0968 , it indicates that there is a significant variation between regions in stunting. Moreover, the values of chi2 $(1)=113.62$ and $p=0.0000$ (see Table 3 ) lead to the rejection of the null hypothesis that the random effect is zero as in the assumption of ordinary logistic regression. From this we can conclude that the random effect at regional level is significantly different from zero. From Table 3 mothers and husband/partner education (secondary and above) significant factors for stunting as compared to their reference categories and age of child in month, place of residence, wealth index, mothers BMI and incidence of diarrhea have significant effect.

\section{The Random Coefficient Model}

Multilevel logistic regression can allow the coefficient of level-one covariates to vary across regions instead of keeping them fixed across regions. Now we are going to see the effect of children level covariates by allowing them to vary randomly across regions. This model contains fixed effects and random effects. The fixed effects are analogous to standard logistic regression coefficients and are estimated directly. The random effects are not directly estimated but are summarized in terms of their estimated variances and covariance. The random effects can take random intercepts (regional effects) and random coefficients (level-one covariates effect). In this section we investigate whether level-one covariates have random effects across regions or they have the same effects across regions. Estimates of this model show that the random slope variances of all included variables except for age children and husband/partner education were approximately zero. This indicates that the effects of age of children and husband/partner education varied across regions whereas the effect of other covariates remained fixed across regions. The results of the random coefficient estimates are given in Table 4 .

In Table 4 the value of $\operatorname{Var}(\operatorname{ageofc} \sim 0)$ and $\operatorname{Var}($ eduahu $\sim$ ) are the estimated variance of age of child in month and husband/partner education respectively. These estimated variances indicated that there is a significant variation in the effect age of child in month and husband/partner education across regions in Ethiopia.

\section{Multilevel logistic regression Model comparison}

Before interpreting multilevel models, we compare the three multilevel logistic regression models (nested models) considered. To do so, deviance, AIC and BIC were used. AIC value of the empty model with random intercept $(\mathrm{AIC}=12125.23)$ is larger than that for the random intercept and fixed coefficient model (AIC $=11371.14)$, which implies that random intercept and fixed slope model is better than the empty model with random intercept in predicting stunting across regions. The significant deviance-based chi-square value for random intercept model indicates that the random intercept and fixed slope model is better than single level multiple logistic regression in stunting across regions as well (see Table 3 and Table 4).

The AIC value of the random coefficient model $(\mathrm{AIC}=11354.56)$ is smaller than the random intercept and fixed coefficient model (AIC $=11371.14)$ implying that random coefficient model is better compared to the random intercept and fixed slope model in describing stunting status (see Table 5) indicating that the random coefficient model is preferred model. Furthermore, the significant deviance-based chi-square value for random coefficient model indicates that the random coefficient model is better than the multiple logistic regressions model in explaining stunting (see Table 4).

Therefore, from the random coefficient model children age group 12-23 and $24+$ had $(\mathrm{OR}=\exp (1.517867))$ 4.562 and $(\mathrm{OR}=\exp (1.745911)) 5.731$ times more likely to be stunted respectively as compared to age group $0-11$ in months controlling for other variables in the model and random effect at level two. Children who reside in rural area had $(\mathrm{OR}=\exp (0.4230424)) 1.527$ times more likely to be stunted as compared to children who reside in urban area controlling for other variables in the model. Children from medium and rich household are $(\mathrm{OR}=\exp (-$ $0.1442489)) 0.866$ and $(\mathrm{OR}=\exp (-0.2214671)) 0.801$ times less likely to be stunted respectively as compared to children from poor household controlling for other variables in the model.

Compared to children with thinness level $(\mathrm{BMI}<18.5)$ mothers, children belonging to normal level (BMI $18.5-24.9)$ and overweight level/obese $(B M I \geq 25)$ mothers were $(\mathrm{OR}=\exp (-0.1763178)) 0.838$ and $(\mathrm{OR}=\exp (-$ $0.5220219)$ ) 0.593 times less likely stunted respectively controlling for other variables in the model. Children who had incidence of diarrhea in the last two weeks are $(\mathrm{OR}=\exp (0.3190488)) 1.376$ times more likely stunted as compared to children who had no diarrhea controlling for other variables in the model. Specifically, children from mothers who had secondary and above educational level are $(\mathrm{OR}=\exp (-0.3770605)) 0.686$ times less likely stunted as compared to children from no education mothers and also children from husband/partner who had secondary and above educational level are $(\mathrm{OR}=\exp (-0.4902556)) 0.612$ times less likely stunted as compared to children 
from no education husband/partner controlling for other variables in the model

An overall evaluation of the multilevel logistic model was assessed using the deviance that is good model is the model that have small value of deviance and also test is done by comparing the deviance of two models by subtracting the smaller deviance from the larger deviance. The difference is a chi-square with the number of degrees of freedom equal to the number of different parameters in the two models. The significance of this chi square indicates that the model is a good fit. Similarly, it was also assessed by using AIC. Based on Table 5 for stunting random coefficient model have a significant deviance chi-square and the value of AIC are less than from the random intercept with fixed slope model and Random Intercept Only Model So, we conclude that the random coefficient model is a good fit.

\section{Discussion}

The results of the study indicate that age of child is one of determinant associated with stunting status of children in Ethiopia. The stunting was higher in children aged greater than 12 months than the age 0-11 groups. This finding is consistent with the studies conducted by Kabubo-Mariara et al. (2006); Shrimpton et al. (2001); Nguyen and Kam., 2008; Alemu Adeba, Sileshi (2014); which revealed a rapid fall in children's height from birth to 59 months; although stunting continues after 24 months and children in the youngest age 0-11 months had significantly lower risk of being stunted, underweight and wasted than children in the older age groups. This could be as a result of weaning and lower breast milk intakes, which make them prone to childhood stunting.

Mother's highest educational level was identified to be the most significant factor to reduce the occurrence of children stunting. The findings of this study showed that there is a significant difference in the status of stunting in children by mothers' educational level. The risk of worse level stunting is significantly higher for children whose mothers have no education and primary education level than children whose mothers have secondary and higher level of education. This finding is consistent with other studies (Nure, Nuruzzaman and Goni, 2011, Semali, I.A.; Tengia-Kessy, 2015; Blessing Jaka Akombi, 2017). They indicated that education improves the ability of mothers to implement simple health knowledge and facilitates their capacity to manipulate their environment including health care facilities, interact more effectively with health professionals, comply with treatment recommendations, and keep their environment clean. Furthermore, educated women have greater control over health choices for their children. This finding also suggests that stunting status was found highest for the children having father's/partner with no education when compared with higher level educated fathers' children (Nguyes and Kam, 2008; Blessing Jaka Akombi, 2017). Place of residence were found to be significant determinants of stunting status in under five children. The analysis also showed that children whose parents reside in rural areas more likely to be stunted when compared to those children whose parents reside in urban areas. This study is similar to the study conducted by Shen et al. (1996), Fotso JC, Kuate-Defo(2005).

The study revealed that under-five children from poor households are at a higher risk of stunting than children from rich households. This finding is similar with studies Woldemariam and Timotewos, (2002); Smith et al., (2005); Alemu Adeba, Sileshi (2014); Loida, Gloria (2017).

Mother's nutritional status significantly influences the stunting status of children. Children from thinness level (BMI<18.5) mother's higher status of stunting as compared to normal level (BMI 18.5-24.9) and overweight (BMI $\geq 25)$ mothers. This finding is consistent with study conducted by Pendael Zephania Machafuko (2013); Semali, I.A.; Tengia-Kessy (2015) which reveals that mother's nutritional status had positive effect indicating that children belong to thinness level $(\mathrm{BMI}<18.5)$ mothers are associated with high probability of stunting

Male children have greater risk of status of stunting than female children Salah E.O. Mahgoub et al., (2006). The result this finding is consistent with these studies but, the covariate genders of a child are insignificant factor for stunting status. This finding also similar with the studies conducted by Salah and Theopi (2006). The result of this study indicates that children who had incidence of diarrhea in the last two weeks are significant factor for stunting as compared to children who had no diarrhea. This study is consistent with the study Alemu Adeba, Sileshi (2014); Blessing Jaka Akombi, (2017).

\section{Conclusion}

Multilevel logistic regression shows that there is heterogeneity or cross-regional variation in stunting. Further this model implies that there exist considerable differences in stunting among regions and a model with a random coefficient is more appropriate to explain the regional variation than a model with fixed coefficients or empty model with random effects. Age of children, region, place of residence, wealth index, mothers BMI, incidence of diarrhea in the last two weeks before survey and mother and husband/partner educational level were found to be significant predictors for stunting. The concerned bodies (government and other stakeholders) have to give different priorities to different children age group, family background in terms of their mothers fathers education level, mother's nutritional status, incidence of diarrhea and fever, household wealth status to control children stunting. Since we have shown that children in rural areas are more likely to be stunted than children in urban areas, special attention should be given for the residence difference. Since there are variations in stunting across 
regions the concerned body should give special attention to regional variation.

Declarations

Authors, declare that this article is our original work and has not been published on any journal.

Ethics approval and consent to participate

Not Applicable

Consent for publication

Not Applicable

Availability of data and material

The analysis in this study is based on data available from the Ethiopian Demographic and Health Survey.

Competing interests

The authors declare that they have no competing interests.

Funding

Not Applicable

Authors' contributions

Hulle Hassen and Kemal Nure generated the idea and designed the study and also doing statistical analysis, editing, reviewing and write up the manuscript. All authors read and approved the final manuscript.

Acknowledgements

Authors acknowledge Madda Walabu University, Bale Robe, Ethiopia.

\section{REFERENCE}

Agresti A. (2002). Categorical data analysis. John Wiley \& Sons, New York, 2nd edition.

Alemu Adeba, Sileshi Garoma, Habtamu Fekadu and Wondu Garoma 2014 Prevalence's of Wasting and its Associated Factors of Children among 6-59 Months Age in Guto Gida District, Oromia Regional State, Ethiopia

Blessing J.Akombi, Kingsley E.Agho, Dafna Merom, John J.Hall and Andre M.Renzaho published January 2017 Multilevel Analysis of Factors Associated with Wasting and Underweight among Children Under-Five Years in Nigeria

Desalegne, Amare, Ayenew Negesse, Baye Tsegaye, Birtukan Assefa, and Birehanu Ayenie.Prevalence of Undernutrition and Its Associated Factors among Children below Five Years of Age in Bure Town, West Gojjam Zone, Amhara National Regional State, Northwest Ethiopia 2016.

De Onis M. WHO child growth standards: length/height-for-age, weight-for-age, weight-for-length, weight-forheight and body mass index-for-age. WHO. 2006.

Ethiopian DHS 2016, Central Statistical Agency report, Addis Ababa, Ethiopia.

Fotso JC, Kuate-Defo B. Socioeconomic inequalities in early childhood malnutrition and morbidity: modification of the household-level effects by the community SES. Health Place 2005;11: 205-25.

Genebo Timotiows and Girma Woldemariam. (2002)“Determinants of Nutritional Status of Women and Children in Ethiopia”. ORC Macro, Calverton, Maryland USA. P. 1-36. (Assessed at http://www.measuredhs. comnutrition.pdf dated May 22nd, 2007).

Hosmer, D. W. and Lemeshow, S. (2000): Applied logistic regression, John Wiley \& Sons, New York, 2nd edition.

Kabubo-Mariara, J., Ndenge, G.K., and Kirii, D.M. (2006). Determinants of Children's Nutritional Status in Kenya: Evidence from Demographic and Health Surveys. Paper Presented at the Centre for the Study of African Economies Conference, University of Oxford, March 17-21, 2006.

Loida María García Cruz, Gloria González Azpeitia, Desiderio Reyes Súarez, Alfredo Santana Rodríguez, Juan Francisco Loro Ferrer and Lluis Serra-Majem: Factors Associated with Stunting among Children Aged 0 to 59 Months from the Central Region of Mozambique

McCullagh P. and Nelder J. A. (1989), Generalized linear models (2nd Ed.). London: Chapman and Hall.

Mussie Alemayehu et al (2014). Nutritional status and associated factors among under-five children, Tigray, Northern Ethiopia.

Alemayehu et al (2014). Nutritional status and associated factors among under-five children, Tigray, Northern Ethiopia.

Nguyen, N.H., and Kam.S. (2008).Nutritional status and the characteristics related to malnutrition in children under-five years of age in Nghean, Vietnam. Journal of preventive medicine and public health, 41(4), 232240.

Nure, A. S., Nuruzzaman, H., Abdul, G. (2011). Mulnutrition of underfive children: Evidence from Bangladesh. Asian Journal of medical sciences 2,113-119.

Pendael Zephinia Machafuko, Geert MOLENBERGHS and Y.C. MUZANILA. Determinants of Nutritional Status Among Children Under Age 5 in Mainland Tanzania. Master Thesis, Universiteit Hasset| Maastrich University, 2013.

Salah, E.O., Malgoub, M. N., Theopi, T.B. (2006). Factors affecting prevalence of malnutrition among children 
under three years of age in Botswana. African Journal of food agriculture nutrition and development $6,1$.

Semali, I.A.; Tengia-Kessy, A.; Mmbaga, E.J.; Leyna, G. Prevalence and determinants of stunting in under-five children in central Tanzania: Remaining threats to achieving Millennium Development Goal 4. BMC Public Health 2015, 15, 1153. [CrossRef] [PubMed]

Shrimpton, R., Victoria, C.G., Onis, M.de., Lima, R.C., Blossner, M., and Clugston, G. (2001). Worldwide Timing of growth faltering: Implications for nutritional interventions. Paediatrics, 107,75-81.

The state of the world's children 2013. Children with disabilities. New York: United Nations Children's Fund; 2013 (http://www.unicef.org.uk/Documents/Publication-pdfs/sowc-2013-children-with disabilities.pdf, accessed 21 October 2014

UNICEF. State of the World's Children Statistical Report. 2015.

Walker SP, Chang SM, Powell CA, Simonoff E, Grantham-McGregor SM. Early childhood stunting is associated with poor psychological functioning in late adolescence and effects are reduced by psychosocial stimulation. J Nutr. 2007;137: 2464-9.

WHO (2000) Global agenda for combating malnutrition: progress report. Geneva, World Health Organization World Health Organization (WHO) Child Growth Standards (2006): http://www.who.Int /childgrowth/en/.

Table 1: stunting and wasting status of children

\begin{tabular}{|l|l|l|}
\hline Stunting status & Frequency & Percent \\
\hline Not stunted & 5891 & 62.9 \\
\hline stunted & 3479 & 37.1 \\
\hline Total & 9370 & 100 \\
\hline
\end{tabular}

Table 2: Result of Variance component model

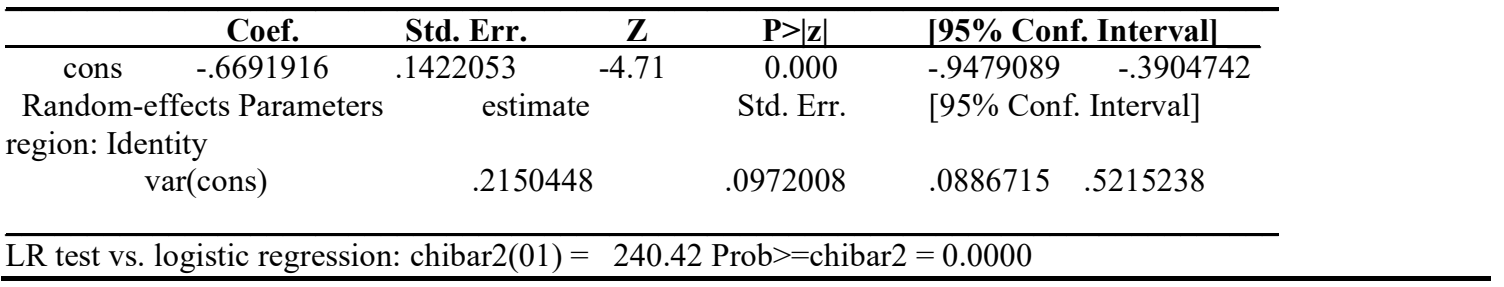

Table 3: Result of Random Intercept and Fixed Slope Model

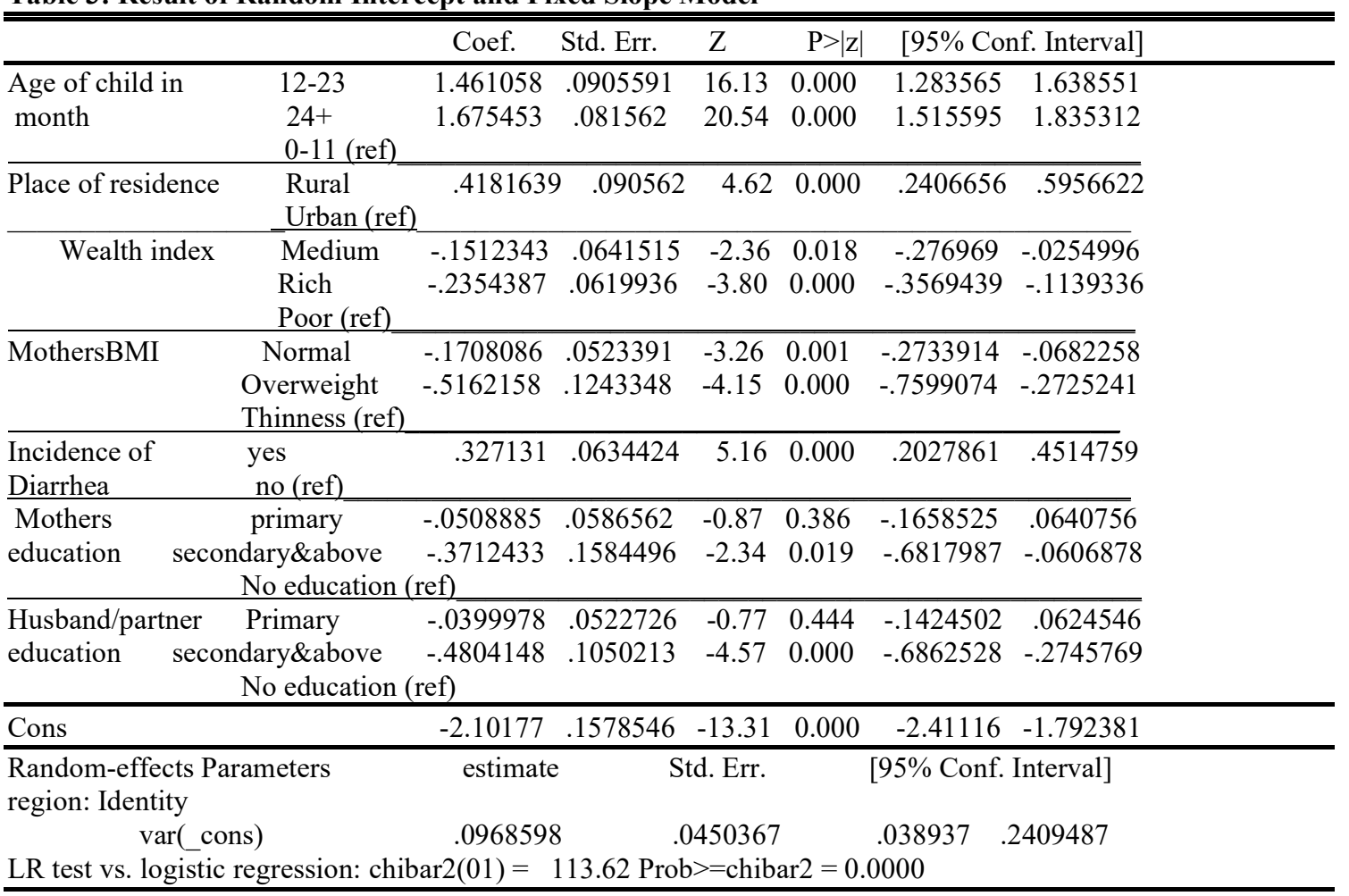


Wald chi2(12) $=009.44$ Prob $>$ chi2 $=0.000$

Table 4: Result of Random Coefficient Model

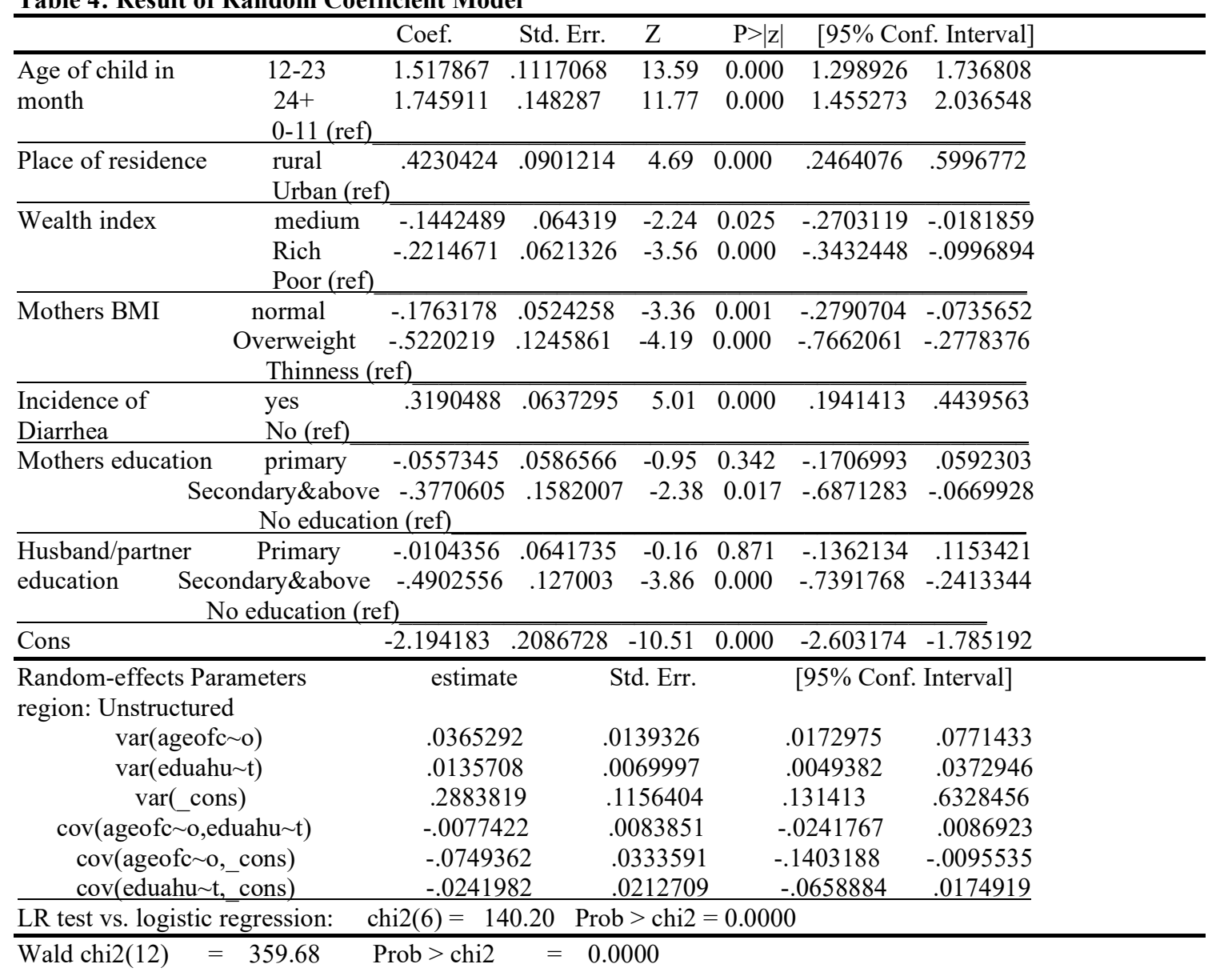

Table 5: Summary of Model comparison

\begin{tabular}{llll}
\hline Model & Log-likelyhood(LL) & -2LL=deviance & AIC \\
\hline Random intercept only Model & -6060.6129 & 12121.226 & 12125.23 \\
Random intercept and fixed slope model & -5671.5683 & 11343.137 & 11371.14 \\
Random coefficient model & -5658.28 & 11316.56 & 11354.56 \\
\hline Assumption: $m 2$ nested in $m 1$ & Prob $>$ chi2 $=0.0000$ & & \\
\hline
\end{tabular}

